\title{
Classification of Cerebral Arteriovenous Malformations and Intranidal Flow Patterns by Color-Encoded 4D-Hybrid-MRA
}

\author{
T. Illies, N.D. Forkert, T. Ries, J. Regelsberger, and J. Fiehler
}

\begin{abstract}
BACKGROUND AND PURPOSE: 4D MRA has been evolving as a noninvasive supplement for DSA. The purpose of this study was to evaluate the feasibility of a newly developed blood flow visualization technique for the classification of cerebral AVMs. We hypothesized that 4D-hMRA allows detection of different flow patterns within the nidus as well as differentiation of feeders and draining veins and has very good agreement with DSA regarding the Spetzler-Martin grade.
\end{abstract}

MATERIALS AND METHODS: Thirty-one consecutive patients with AVMs were evaluated by using 4D-hMRA and DSA by 2 blinded raters. Rating criteria included Spetzler-Martin score and other morphologic variables together with a new scale for 3 intranidal flow patterns (homogeneous $=1$, unidirectional $=2$, heterogeneous $=3$ ).

RESULTS: The Spetzler-Martin grades were rated different from DSA in 5 cases by rater 1 and in 3 cases by rater 2 with an excellent interrater reliability of $\kappa=0.96$ (4/31, 1 by size and 3 by drainage). Each reader missed 5 feeders on 4D-hMRA. Draining veins were distinguished in the temporal course in 7 on DSA but in 8 and 12 on 4D-hybrid-MRA (raters 1 and 2 respectively), with $\kappa=0.79$. A type 1 intranidal flow pattern was recognizable in 9 (30\%) patients; type 2, in 19 (60\%); and type 3, in $3(10 \%)$.

CONCLUSIONS: 4D-hMRA allows reliable Spetzler-Martin grading and detection of brain arteriovenous malformation feeding arteries and draining veins, with the drawback that for small vessels DSA is still needed. Draining veins might even be detected with higher sensitivity than on DSA. Discrimination of different intranidal flow patterns is possible, but their relevance for hemorrhage risk assessment and therapy planning requires further study.

ABBREVIATIONS: 4D-hMRA = 4D-hybrid-MRA; ACA = anterior cerebral artery; PCA = posterior cerebral artery; SM = Spetzler Martin; TOF = time of flight; TREAT $=$ time-resolved echo-shared angiographic technique

T ime-resolved (4D) MR angiography enables the examination of arterial, venous, and mixed contrast phases similar to that of conventional DSA. Subsecond temporal resolution and an acceptable spatial resolution of today's widely available 4D-MRA techniques made these sequences suitable for clinical routine and scientific purposes, albeit the temporal and spatial resolution is still inferior to that on DSA. However, MRA is noninvasive, and effort is increasing to replace DSA in the clinical routine. Besides lower invasiveness, 4D-MRA offers potential advantages over

Received December 29, 2011; accepted after revision February 29, 2012.

From the Departments of Diagnostic and Interventional Neuroradiology (T.I., T.R., J.F.), Computational Neuroscience (N.D.F.), and Neurosurgery (J.R.), University Medical Center Hamburg-Eppendorf, Hamburg, Germany.

Please address correspondence to Till Illies, MD, Department of Neuroradiology, University Medical Center Hamburg-Eppendorf, Martinistraße 52, 20246 Hamburg, Germany; e-mail: t.illies@uke.uni-hamburg.de

O-- Indicates open access to non-subscribers at www.ajnr.org

E Indicates article with supplemental on-line table.

http://dx.doi.org/10.3174/ajnr.A3204 conventional DSA. First, tomographic imaging of blood flow within the nidus can be achieved. Second, in contrast to 3D rotational angiography, real 4D blood flow visualization from any view angle can facilitate the understanding of difficult vascular anatomy. Third, the depiction of all feeding vessels at the same time offers additional information over separate acquisitions of discrete vessel territories in different DSA injections.

4D-MRA might improve the understanding of vascular malformations such as cerebral AVMs because information about blood flow direction and velocity is essential for understanding the underlying hemodynamics and pathology. Very good agreement of conventional 4D-MRA and DSA regarding the Spetzler-Martin grading of brain AVMs has been shown in several studies, with a spatial resolution of up to $1.1 \times 1.1 \times 1.1 \mathrm{~mm}^{3}$ and high temporal resolution of up to $680 \mathrm{~ms} /$ frame. ${ }^{1-4}$ Moreover, it was shown that depiction of feeding arteries and draining veins with $4 \mathrm{D}$-MRA is only slightly inferior to that in DSA, with a lack of sensitivity for small vessels. This could be improved by additional arterial spin-labeling perfusion imaging $^{1,5}$ but still did not reach the accuracy of DSA. 

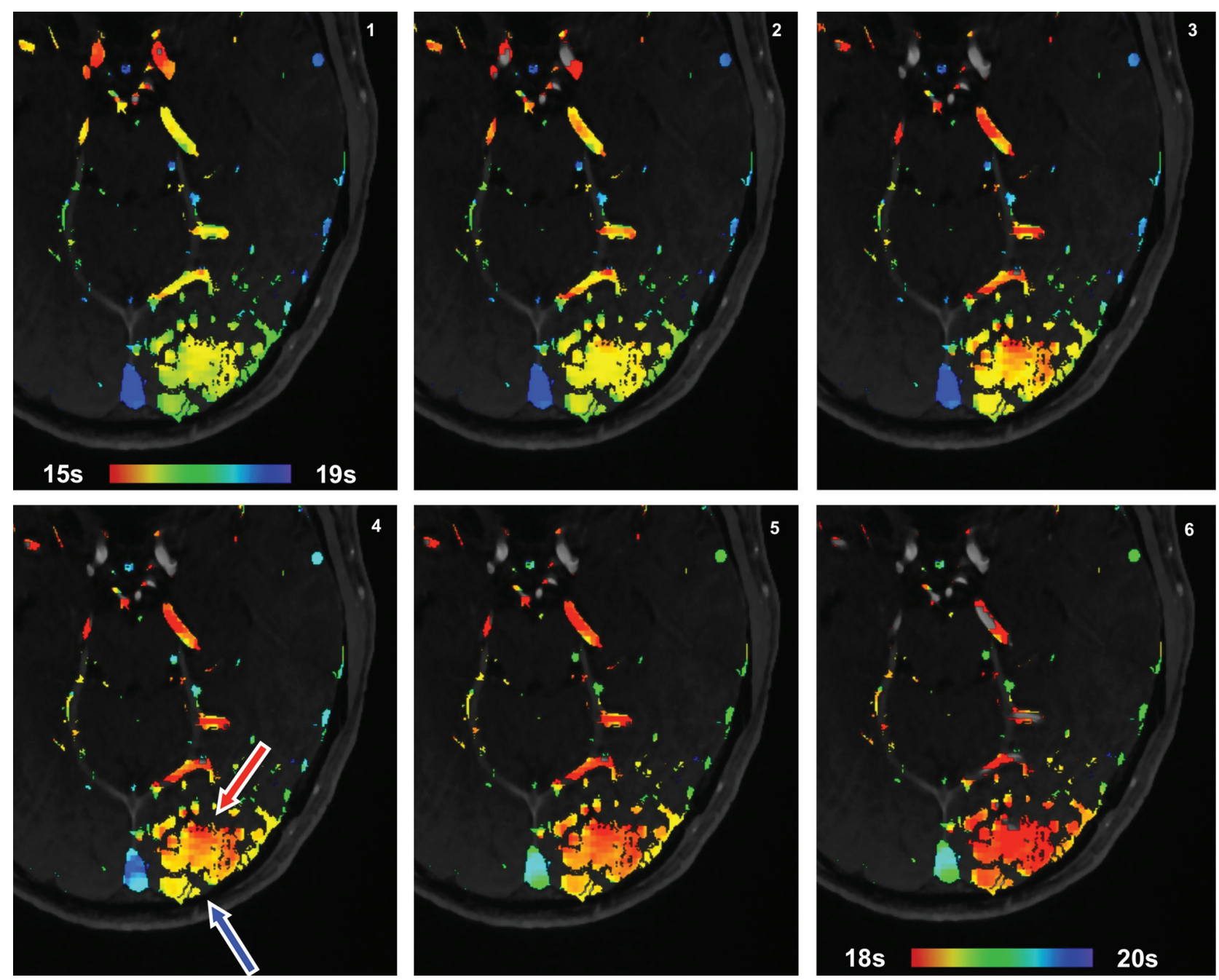

FIG 1. Patient 5. Homogeneous nidus perfusion (type 1). Inflow time point range is set from 15 to 19 seconds in the first image to $18-19$ seconds in the last to generate the effect of blood flow. Corresponding to the inflow from the PCA and outflow into the transversal sinus, the nidus is perfused in an anterolateral-to-posteromedial direction.

Recently, we proposed a 4D-hMRA visualization technique combining blood flow information from 4D-MRA with the anatomic information from high-resolution 3D MRA datasets. ${ }^{6}$ Contrast agent arrival times within the cerebral vasculature, which are inversely correlated to blood flow velocity, are color-encoded and superimposed on a 3D cerebrovascular model extracted from 3D TOF MRA.

This way, we achieved high temporal (virtual analog $0.1 \mathrm{sec}-$ ond) and spatial resolution $\left(0.47 \times 0.47 \times 0.5 \mathrm{~mm}^{3}\right)$ by using widely available MR imaging sequences. Hemodynamic classification of cerebral AVMs, including and beyond the anatomic grading scheme of the Spetzler-Martin classification, ${ }^{7}$ was expected. We hypothesized that this $4 \mathrm{D}-\mathrm{hMRA}$ method reveals very good agreement with DSA on Spetzler-Martin classification, allows temporal discrimination of draining veins, and enables the detection of different flow patterns within the nidus.

\section{MATERIALS AND METHODS}

\section{Patients}

From April 2007 to September 2008, 31 consecutive patients with an AVM were enrolled in this study. The inclusion criterion was an AVM diagnosed by conventional digital subtraction angiography. Informed consent was obtained from all patients. The study was approved by the local ethics committee (No. 2706/2005). Fifteen patients were female, and 16 were male with a median age of 44 years (range, 15-72 years). Nine AVMs presented with hemorrhage (with a mean delay of 263 days between hemorrhage and MR imaging measurement); 5 , with seizures; 6 , with headache; 1 , with a focal neurologic deficit; and 7 AVMs were detected incidentally.

\section{Imaging}

Conventional DSA (AlluraXper; Philips Healthcare, Best, the Netherlands) with 3 images per second, with an FOV of $22 \times 22$ $\mathrm{cm}$ and a matrix of $1024 \times 1024$ pixels (resolution of approximately $0.2 \times 0.2 \mathrm{~mm}$ ) in standard projections with injections in both ICAs and at least 1 vertebral artery was performed in all patients. In 12 patients, MR imaging measurements were obtained on a 1.5T Sonata and in 19 on a 3T Trio scanner (Siemens, Erlangen, Germany), by using an 8-channel phased-array head coil. Among others, we used the following sequences:

As a $4 \mathrm{D}-\mathrm{MRA}$, the $\mathrm{TREAT}^{8}$ sequence was used, which was 

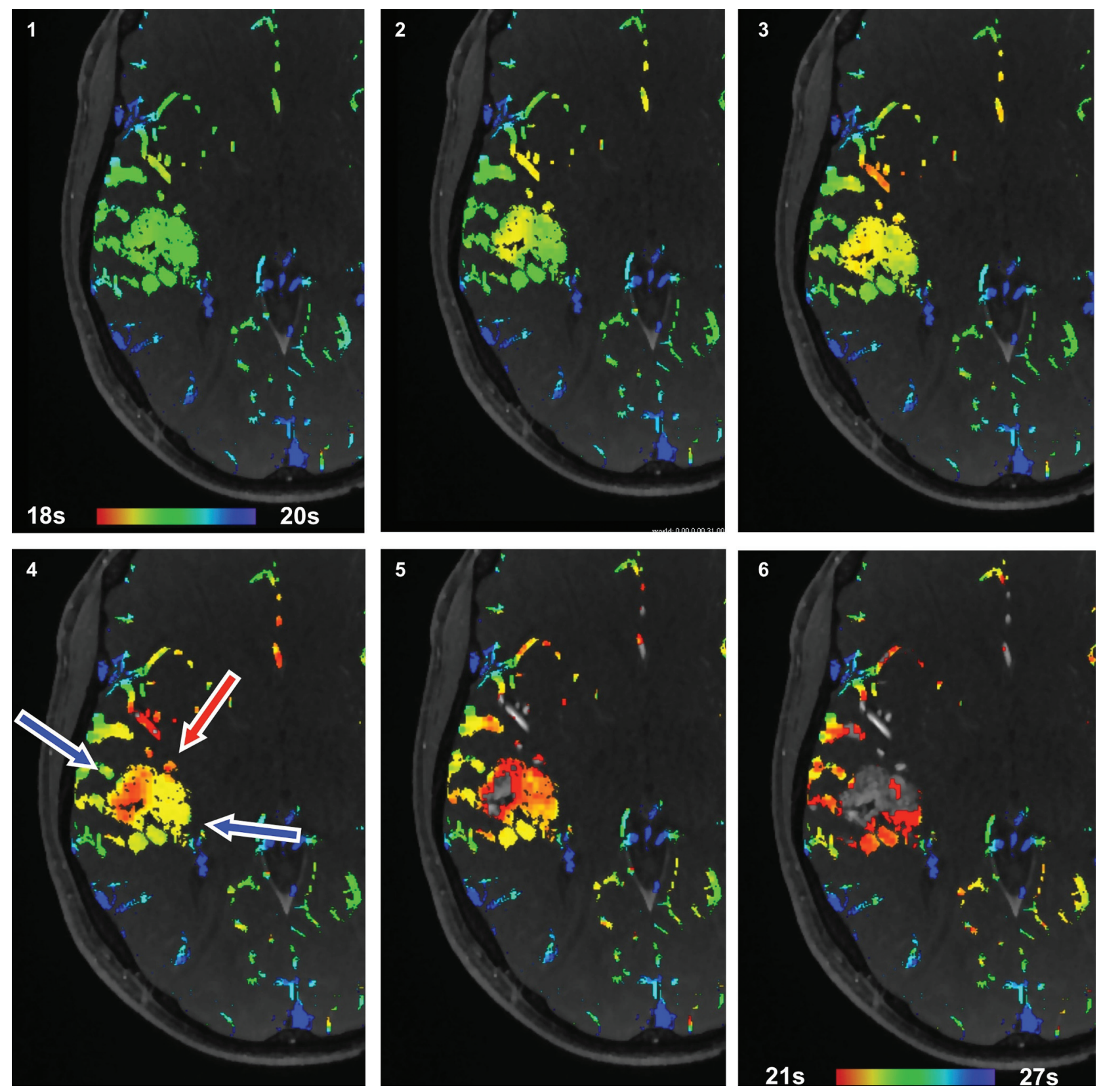

FIG 2. Patient 9. Inhomogeneous nidus perfusion (type 2). Inflow time point setting from 18 to 20 seconds in the first image to $21-27$ seconds in the last image to generate the effect of blood flow. Diversion of the central inflow into the nidus can be observed to the lateral and medial sides.

implemented by using a 3D fast low-angle shot sequence with TE/TR of $0.69 / 2.54 \mathrm{~ms}, 20^{\circ}$ flip angle, in-plane image resolution of $1.875 \mathrm{~mm}^{2}$, a section thickness of $5 \mathrm{~mm}$, and an FOV of $230 \times$ $172.5 \times 80 \mathrm{~mm}$. Parallel imaging with a generalized autocalibrating partially parallel acquisition factor of 2 was applied. Contrast injection was performed by intravenous pump injection of $20-\mathrm{mL}$ gadobenate dimeglumine (MultiHance; Bracco Altanta Pharma, Konstanz, Germany) at $4 \mathrm{~mL} / \mathrm{s}$, followed by $20-\mathrm{mL}$ isotonic saline. This imaging technique allowed the acquisition of one $3 \mathrm{D}$ dataset in 0.5 seconds.

A 3D TOF $^{9}$ MRA was implemented with a magnetization transfer saturation pulse, a TR of $36 \mathrm{~ms}$, a TE of $6 \mathrm{~ms}$, a flip angle of $25^{\circ}$, five slabs with 40 partitions each, an in-plane resolution of $0.47 \times 0.47 \times 0.5 \mathrm{~mm}^{3}$, and an FOV of $150 \times 200 \mathrm{~mm}^{2}$.

\section{D-Hybrid MRA Blood Flow Visualization}

The 3D TOF and 4D TREAT sequences are the basis for the 4Dhybrid blood flow visualization used in this study. Briefly described, the cerebrovascular system is automatically extracted from the 3D TOF image sequence by using methods described by Forkert et al. ${ }^{10-12}$ This segmentation is then used for generation of a $3 \mathrm{D}$ surface model by using the Marching Cubes algorithm. In a following step, the contrast signal curves from the 4D TREAT image sequence are analyzed in terms of voxelwise estimation of the TTP by using reference-based linear curve fitting analysis. ${ }^{12}$ The calculated TTP maps are then coregistered to the TOF dataset. ${ }^{6}$ Finally, the registered TTP map is masked with the vessel segmentation and can be viewed color-coded together with the TOF dataset, allowing display of the intranidal flow. The color 

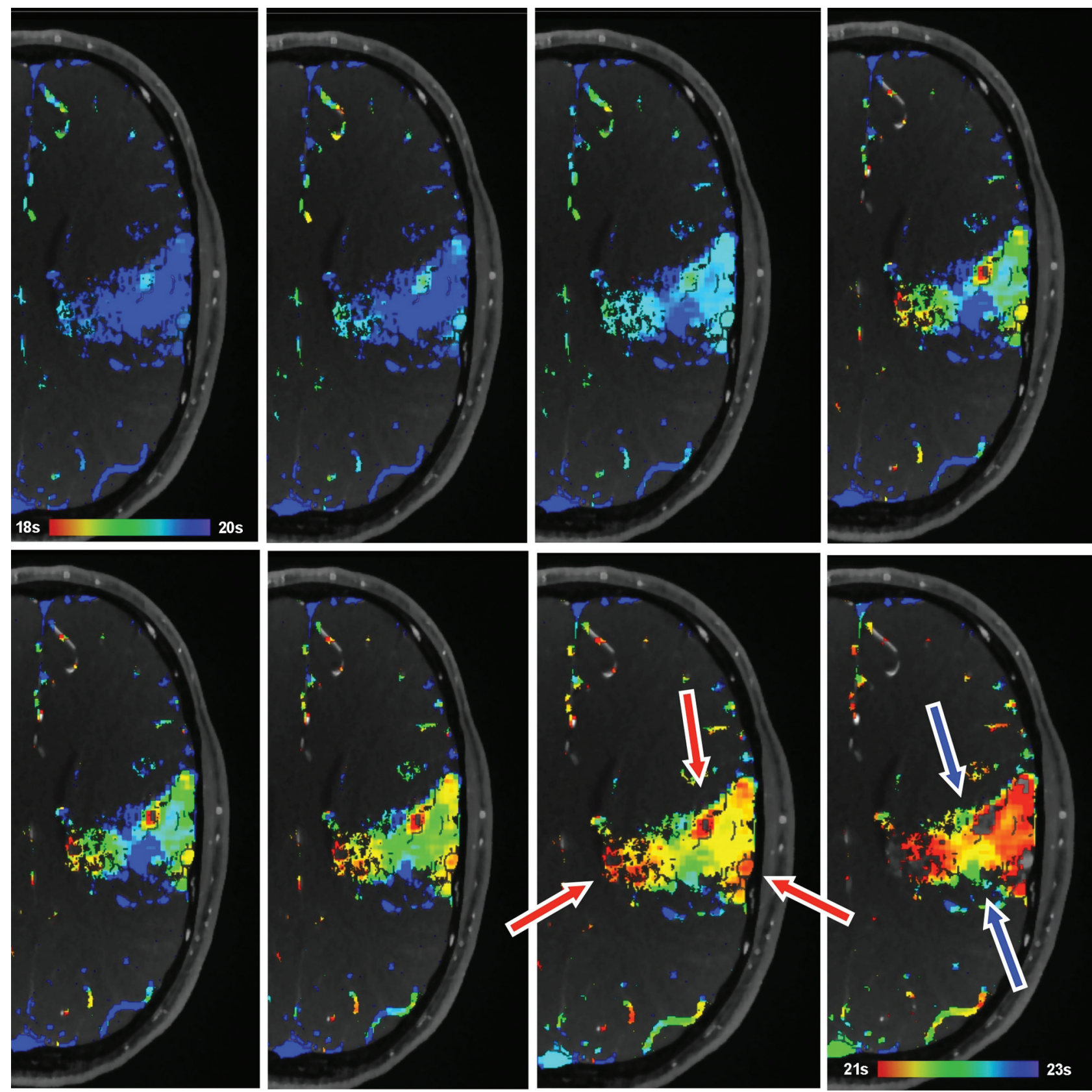

FIG 3. Patient 6. Inhomogeneous nidus perfusion (type 3). Inflow time point setting from 18 to 20 seconds in the first image to $21-23$ seconds in the last image to generate the effect of blood flow. Corresponding to different feeding territories, inflow is seen in the paraventricular region and in the MCA territory anteriorly and laterally (red arrows) with multidirectional flow diversion toward drainage (blue arrows).

range of the TTP representation of interest for a certain vessel section can also be adjusted interactively by using a similar window-leveling technique. Furthermore, the extracted TTP information can be mapped onto the $3 \mathrm{D}$ cerebrovascular surface model where it can also be visualized color-coded as well as interactively.

\section{Image Analysis}

DSA examination was reviewed by 1 neuroradiologist (J.F. with $>12$ years of experience in neuroradiology) in random order, blinded to patient history and MR imaging examinations. DSA was rated for feeding arteries, number of draining veins, and elo- quent location of the nidus, deep venous drainage, Spetzler-Martin score, and sequence of draining veins.

4D-hMRA was read independently by 2 neuroradiologists (T.R. with $>10$ years of experience and T.I. with $>6$ years of experience in neuroradiology) by using the software system Antonia R. These readers were also blinded to DSA and patient history. Readers classified the AVMs according to Spetzler-Martin graduation (deep drainage, eloquent location, and size of the nidus), number of feeding arteries and draining veins, and whether the sequence of draining veins could be distinguished. Classification of the intranidal flow pattern was available for reader 2 only. Three intranidal flow patterns were discernible: Type 1 is a homo- 

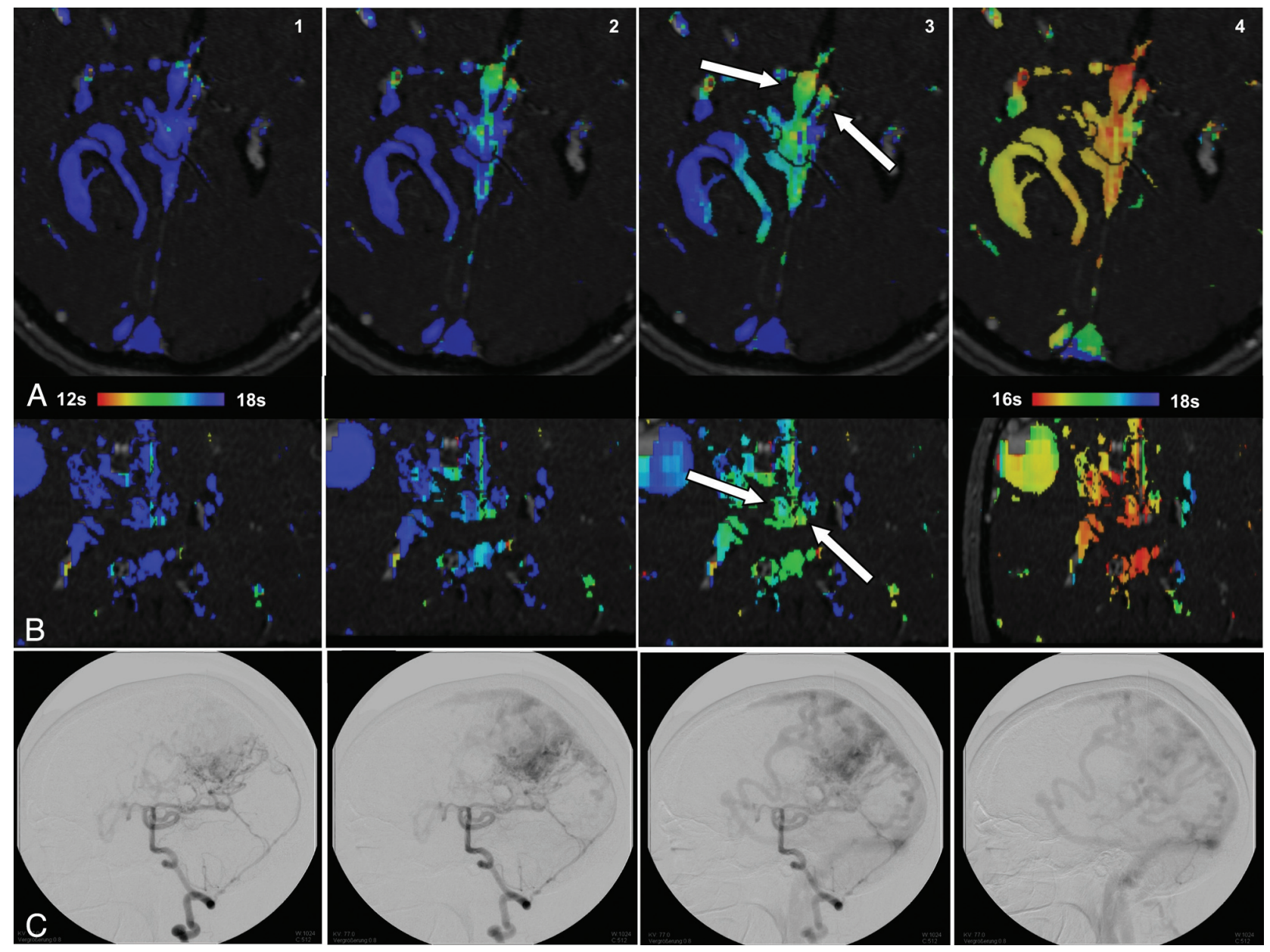

FIG 4. Patient 23. Axial $(A)$ and coronal $(B)$ color-encoded images show the right (ipsilateral to the AVM) internal cerebral vein dilated with symmetric inflow time points. Note the early inflow in the deep venous system in comparison with the confluens sinuum (column 3). On DSA, a deep venous drainage is not seen $(C)$. Also note the huge aneurysmal dilation of cortical drainage with relatively late filling from its medial portion.

geneous unidirectional flow (Fig 1). Type 2 flow pattern is heterogeneous with 2 focal areas of either early or late inflow time points indicating a single branching of flow (Fig 2). Type 3 flow pattern has $>2$ focal "peaks" or "dips" so that the direction of the intranidal flow cannot be determined and appears chaotic (Fig 3).

\section{Statistics}

The Kendall W coefficient of concordance was used to compare the interrater reliability between readers 1 and 2 for 4D-hybridMRA visualizations and between each reader and DSA. Coefficients of $0.5-0.8$ were considered good agreement, and above 0.8 , excellent agreement. $P$ values $<.05$ were considered statistically significant. Statistics were calculated by using R 2.12.2.

\section{RESULTS}

\section{Imaging}

Cranial DSA as well as 3D and 4D-MRA images were available for all patients with sufficient quality for diagnostic and computeraided image analysis purposes. Datasets at $3 \mathrm{~T}$ and $1.5 \mathrm{~T}$ were acquired with the same temporal and spatial resolution to obtain the same datasets for postprocessing. The 1.5T datasets showed some artifacts not seen on 3T: Three datasets had time-invariant hyperintensities of arteries that run in-plane and were localized at the margins of the FOV. Two other datasets revealed an alternating baseline superimposed on the signal-intensity curves. However, approximation of the signal-intensity curves allowed production of visualization results for $1.5 \mathrm{~T}$ datasets that are comparable with those for $3 \mathrm{~T}$ for visual assessment as conducted in this study.

\section{Spetzler-Martin Score}

Nidus Location. In 19 of 31 patients, the nidus was located in an eloquent brain region by both readers on $4 \mathrm{D}$-hMRA ( $100 \%$ agreement, Kendall $W=1$ ). This was in total congruence with DSA (100\% agreement, Kendall $W=1$ ).

Deep Drainage. Twenty-one of 31 AVMs showed drainage into the deep venous system on DSA. Reader 1 classified 23 (Kendall $W=0.85$ ) (4 incorrectly: 3 more and 1 less than on DSA), and reader 2 classified 24 patients (Kendall $W=0.89$ ) ( 3 incorrectly: 3 more than on DSA) as having a deep drainage in $4 \mathrm{D}$-hybridMRA. Interrater reliability was good with disagreement in 3 AVMs (Kendall $W=0.87$ ).

Nidus Size. Reader 1 measured the nidus with a mean size of $37.5 \pm 15.5 \mathrm{~mm}$ (according to SM grade of $1.7 \pm 0.6$ ), and reader 2 , with $36.1 \pm 15.5 \mathrm{~mm}$ (according SM grade of $1.7 \pm 0.7$ ) with an 

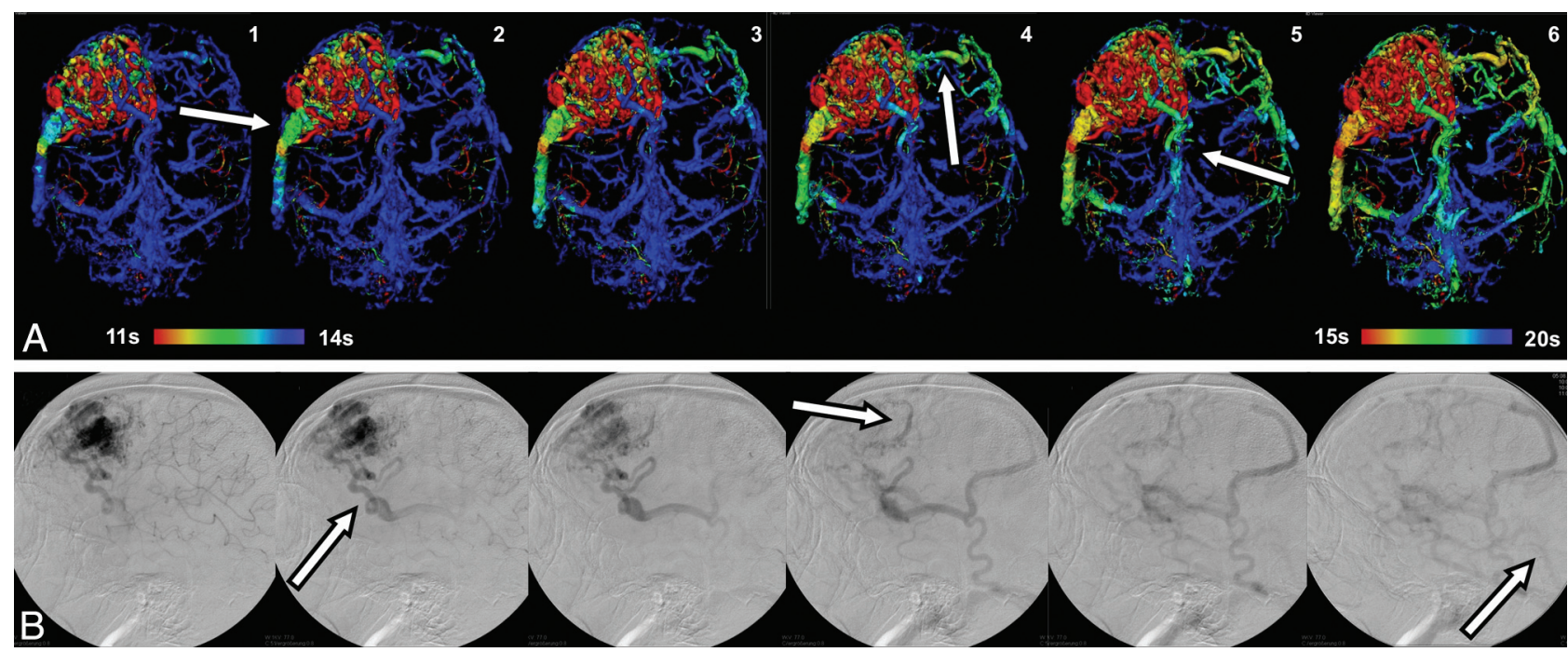

FIG 5. Patient 4. A, Two dilated superficial draining veins can be seen, which drain early into the transverse sinus and superior sagittal sinus (pictures 1 and 4). On picture 5, a dilated vein with delayed drainage into the internal cerebral veins is seen. B, DSA shows faint and retarded drainage into the deep venous system (picture 5).

inter-rater reliability of Kendall $W=0.98$ for the SM grade. In DSA, nidus size was averaged with $36.0 \pm 14.5 \mathrm{~mm}$ (according to SM grade of $1.7 \pm 0.7$ ) (Kendall $W=0.98$ for reader 1 and Kendall $W=1$ for reader 2).

Spetzler-Martin Score. The total Spetzler-Martin score was determined with 4 discordant results between both raters on 4DhMRA. Rater 1 had 5 discordances with conventional DSA (Kendall $W=0.94$ ), and rater 2 had 3 discordances (Kendall $W=$ 0.97). Spetzler-Martin scores were $3.0 \pm 0.9$ for DSA, $3.1 \pm 0.9$ for reader 1 , and $3.1 \pm 1.0$ for reader 2 with an interrater reliability of Kendall $W=0.96$ (4/31 differences in 4D-hybrid-MRA visualizations, 1 by size and 3 by drainage) (On-line Table).

\section{Feeders and Hemodynamic Evaluation}

Feeders. In 6 patients, the full number of feeders was missed on 4D-hMRA in comparison with DSA. Readers 1 and 2 each missed 5 feeders. All were $\leq 2 \mathrm{~mm}$ in diameter.

Discrimination of Draining Veins. In 7 AVMs, it was possible to distinguish the sequence of draining veins on DSA images. In the 4D-hMRA, reader 1 classified 8 (3 different from DSA, Kendall $W=0.87$ ) and reader two, 12 draining veins (7 different from DSA, Kendall $W=0.76$ ) as distinguishable, giving an interrater reliability of Kendall $W=0.79$ with 6 differences in 4D-hybrid-MRA.

Intranidal Flow Pattern. A type 1 pattern was recognizable in 9 (30\%) AVM nidi, type 2 in 19 (60\%), and type 3 in 3 (10\%). The type of nidus perfusion was not related to the rupture status of the AVM. Ruptured AVMs had a mean nidus perfusion type of $1.77 \pm$ 0.76 , and unruptured, a median nidus perfusion type of $1.81 \pm$ $0.59(P=.66)$ (On-line Table).

\section{DISCUSSION}

4D MR angiography and lately also $4 \mathrm{D}-\mathrm{CTA}^{13}$ have been evolving in clinical routine as noninvasive supplements to DSA. We combined the benefits of 3D TOF MRA and 4D contrast-enhanced MRA to increase spatial resolution and obtain a virtual temporal resolution of up to 0.1 -second inflow time points, which serve as a measure of blood flow velocity, are color-coded, and can be adjusted interactively, giving the impression of a contrast bolus flowing through the vessels. This way, arterial, venous, and mixed-phase images can be obtained. For this study, data were acquired in a clinical routine setting at $1.5 \mathrm{~T}$ and $3 \mathrm{~T}$. Due to the curve-fitting algorithm of the postprocessing, the inferior field strength at $1.5 \mathrm{~T}$ did not influence visualization results compared with $3 \mathrm{~T}$ datasets.

We found very good agreement for the Spetzler-Martin classification between DSA and 4D-hMRA. The agreement is in line with previous studies on 4D-MRA. ${ }^{2,14-16}$ In our study, the differences in Spetzler-Martin scores are attributed to the definition of the drainage pattern and nidus size measurement, while the nidus was located equally well in both examinations. Classification of nidus size and location is intuitively superior with tomographic imaging compared with DSA. In our study, the 3 standard planes were used for size measurements instead of angulated planes, which might produce more precise diameters, so that interrater agreement was reduced. In only 1 patient (patient 27), a difference in size measurement caused a different Spetzler-Martin grade because results were above and below the cutoff value of $3 \mathrm{~cm}$.

Interrater reliability and accordance with DSA regarding depiction of draining veins were excellent for both readers, even though variability was somewhat higher and total agreement was not achieved as reported in the previous studies on 4D-MRA. On 4D-hMRA, more AVMs were seen to drain into the deep system than on DSA. We believe that this is a true advantage of 4D-MRA and was caused by a better depiction of draining veins in tomographic images with inflow-time point encoding. For example, patient 23 has a right parietal AVM fed by ACA, PCA, and MCA branches that angiographically drain into the superior sagittal sinus (Fig 4). On transversal and coronal views of the 4D-hMRA, the ipsilateral internal cerebral vein is dilated as an effect of its overload as a draining vein. An earlier inflow with respect to the 

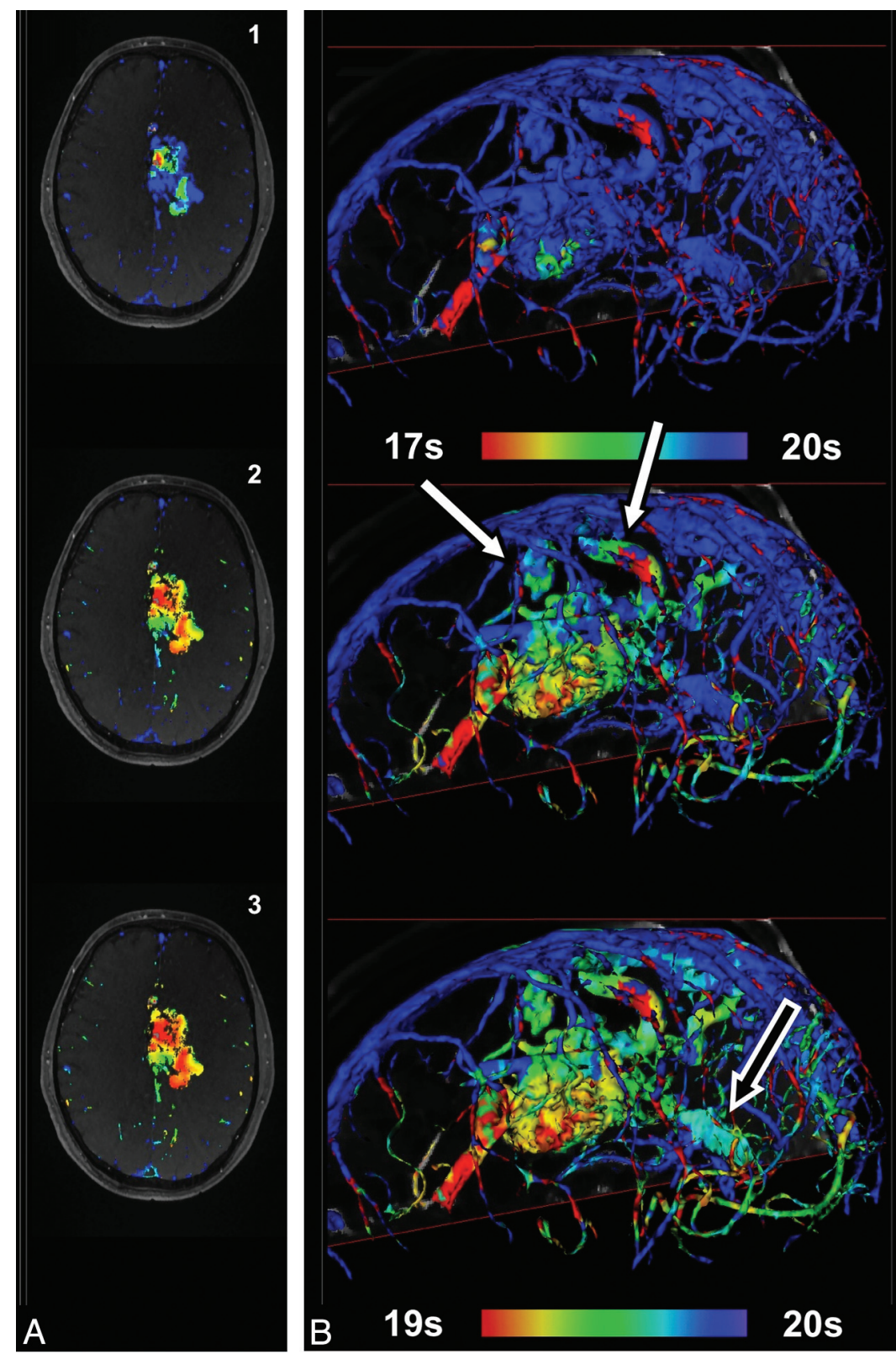

2
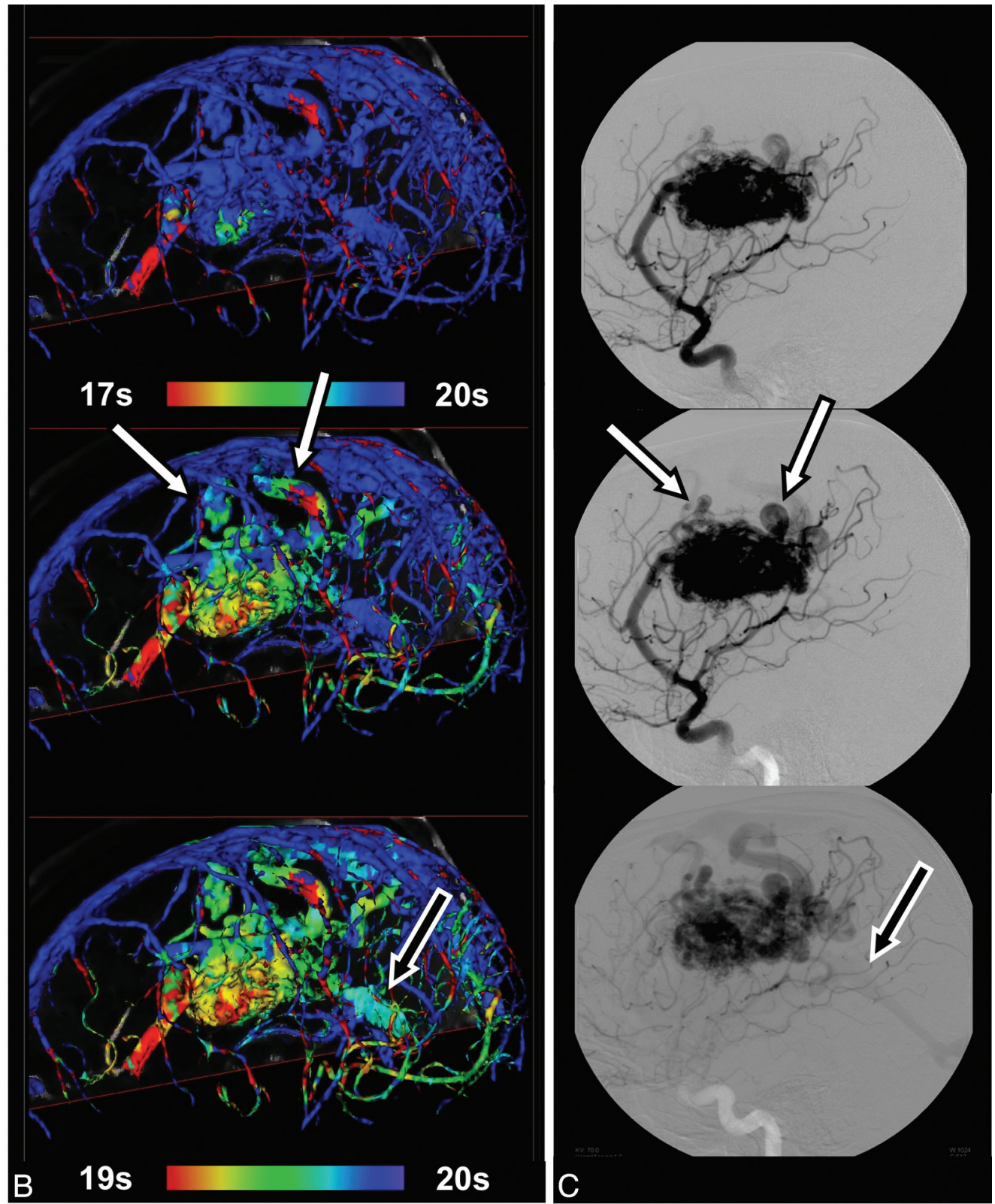

FIG 6. Patient 19. Brain arteriovenous malformation fed by the ACA and PCA. Initial drainage into veins draining into the superior sagittal sinus (white arrows), while the posteromedial part of the nidus begins to drain later into the vein of Galen ( $B$ and $C$, single black arrow). Corresponding inhomogeneous intranidal flow pattern with a late inflow time point in the posteromedial part of the AVM nidus (type 2 ) ( $A$ ) is seen.

contralateral internal cerebral vein is not seen, though. This might be due to a relatively small difference of inflow time points, possibly exceeding the temporal resolution of the 4D-hMRA. An explanation for the poor conspicuity on DSA might be that only a small fraction of blood drains through the internal cerebral vein, resulting in weak attenuation of the $\mathrm{x}$-rays.

Another example is patient 4 with a right frontal AVM that drains superficially into the straight superior and transverse sinuses, which can be well seen on DSA (Fig 5). The delayed deep drainage into the internal cerebral vein is only faint on DSA and can easily be overlooked. On 4D-hMRA, this deep drainage is seen as a dilated vein, which contrasts with the other venous structures due to its early filling in the late arterial phase as a sign of arteriovenous shunt surgery, in effect offering a higher sensitivity. This is also a good example of the ability to differentiate the sequence of the draining veins with 4D-hMRA. Here, interrater reliability and accordance with DSA are also good. Again, the sequences of more draining veins could be distinguished better with 4D-hMRA than with DSA (Figs 5 and 6). This might, in part, be due to the rather slow rate of 3 frames/second that we use for DSA. Higher frame rates might allow differentiation of the sequences of more draining veins but with the trade-off of higher radiation exposure.

The major drawback of 4D-hMRA lies in the depiction of small vessels. This can be seen in the cases in which the sequences of draining veins were not successfully distinguished and can be ascribed to vessels with a diameter smaller than $2 \mathrm{~mm}$ as well as the superior spatial resolution of DSA. The same is true for the depiction of feeding arteries, which is very good in vessels greater than $2 \mathrm{~mm}$ but inferior to DSA in arteries smaller than that. Small vessel size is a problem also encountered in previous studies. 
There, sensitivity could be improved by additional arterial spinlabeling perfusion imaging, with the drawback that only feeders supplying a different vascular territory could be detected. For a reliable detection of all feeding arteries, including vessels smaller than $2 \mathrm{~mm}$, DSA still is needed in addition to 4D-MRA.

As the central anatomic structure, the nidus plays an essential role in the rupture of an AVM and for embolization. Its investigation has only rarely been pursued. 4D-hMRA offers very good insight into flow patterns within the nidus, so fistulas, compartmentation, and "main routes" between feeders and veins are well displayed. Because, in contrast to DSA, all feeding and draining vessels are depicted at the same time, the contribution of each single vessel to the whole nidus perfusion and its main transit routes can be analyzed and related to each other. Complex intranidal flow patterns (type 3, see below), however, can impede this analysis. Here, superselective DSA with a display of each single feeder is an advantage. In color-encoded planar reconstructions of 4D-hMRA, different types of blood flow through the nidus are discernible and can be classified into 3 types: first, a homogeneous flow in 1 direction without branching, being consistent with a major single inflow and a major single outlet as seen in patient 5 (Fig 1). Perfusion types 2 and 3 show a heterogeneous distribution of inflow time points. In type 2, there is a single branching either of 1 inflow point to 2 outflow points or vice versa as seen in patient 9 (Fig 2). Here, early arterial inflow is seen from an MCA feeder in the middle of the convolute. The blood is drained to both sides, laterally and medially, being consistent with a branching of the blood stream to the drainage veins, which are located here.

More complex inflow-time point distributions that exhibit multiple stream debranchings are seen in patient 3 (Fig 3). This flow pattern cannot be explained by only 1 or 2 relevant feeders or draining veins. In Fig 3, early inflow is seen medially, anterolaterally, and posterolaterally. Only delayed inflow is observed in the middle anteriorly and posteriorly, so the nidus is compartmented. In our patient cohort, type 2 was the prevailing flow pattern and was prevalent in almost two-thirds of the patients followed by a homogeneous flow (type 1) and a type 3 pattern. Previously ruptured AVMs do not differ in the type of intranidal flow pattern; also the potential role of different flow patterns, for example in prediction of AVM-related rupture, remains unclear. However, complex flow patterns might indicate a higher probability of turbulence. Turbulent flow increases vessel wall stress and is thought to be associated with AVM rupture. There is a need for a prospective study design to validate this hypothesis with respect to these intranidal perfusion patterns.

\section{CONCLUSIONS}

4D-hMRA allows reliable detection of brain arteriovenous malformation feeding arteries and draining veins with a diameter of $>2 \mathrm{~mm}$. Draining veins might even be detected with higher sensitivity than with DSA with the drawback that for smaller vessels, DSA is still needed. Spetzler-Martin grades can be obtained reliably. With its unique visualization of intranidal flow patterns, 4D-hMRA represents a promising addition to DSA imaging.
Disclosures: Jan Regelsberger-UNRELATED: Payment for Lectures (including service on Speakers Bureaus): University Medical Center Hamburg, Comments: neurosurgical education. Jans Fiehler-RELATED: German Research Fund (DFG), ${ }^{*}$ Comments: governmental funding; UNRELATED: Board Membership: European Society for Minimally Invasive Neurological Therapy (ESMINT), European Society for Neuroradiology (ESNR), Berufsverband Deutscher Neuroradiologen (BDNR), Deutsche Gesellschaft für Neuroradiologie (DGNR), Consultancy: Stryker, Codman, Grants/ Grants Pending: DFG;* Payment for Lectures (including service on Speakers Bureaus): ev3, Stryker, Codman, Philips, Siemens, Bayer. ${ }^{\star}$ Money paid to the institution.

\section{REFERENCES}

1. Kukuk GM, Hadizadeh DR, Boström A, et al. Cerebral arteriovenous malformations at 3.0T: intraindividual comparative study of 4DMRA in combination with selective arterial spin labeling and digital subtraction angiography. Invest Radiol 2010;45:126-32

2. WarrenDJ,HoggardN,WaltonL, etal.Cerebralarteriovenousmalformations: comparison of novel magnetic resonance angiographic techniques and conventional catheter angiography. Neurosurgery 2001;48:973-82, discussion 982-83

3. Hadizadeh DR, Falkenhausen von M, Gieseke J, et al. Cerebral arteriovenous malformation: Spetzler-Martin classification at subsecond-temporal-resolution four-dimensional MR angiography compared with that at DSA. Radiology 2008;246:205-13

4. Nishimura S, Hirai T, Sasao A, et al. Evaluation of dural arteriovenous fistulas with $4 \mathrm{D}$ contrast-enhanced MR angiography at 3T. AJNR Am J Neuroradiol 2010;31:80-85

5. Xu J, Shi D, Chen C, et al. Noncontrast-enhanced four-dimensional MR angiography for the evaluation of cerebral arteriovenous malformation: a preliminary trial. J Magn Reson Imaging 2011;34:1199-205

6. Forkert ND, Säring D, Handels H. Automatic analysis of the anatomy of arteriovenous malformations using 3D and 4D MRA image sequences. Stud Health Technol Inform 2010;160(pt 2):1268-72

7. Spetzler RF, Martin NA. A proposed grading system for arteriovenous malformations. J Neurosurg 1986;65:476-83

8. Fink C, Ley S, Kroeker R, et al. Time-resolved contrast-enhanced three-dimensional magnetic resonance angiography of the chest: combination of parallel imaging with view sharing (TREAT). Invest Radiol 2005;40:40-48

9. Davis WL, Warnock SH, Harnsberger HR, et al. Intracranial MRA: single volume vs. multiple thin slab $3 \mathrm{D}$ time-of-flight acquisition. J Comput Assist Tomogr 1993;17:15-21

10. Forkert ND, Säring D, Fiehler J, et al. Automatic brain segmentation in time-of-flight MRA images. Methods Inf Med 2009;48:399-407

11. Forkert ND, Schmidt-Richberg A, Fiehler J, et al. Fuzzy-based vascular structure enhancement in time-of-flight MRA images for improved segmentation. Methods Inf Med 2011;50:74-83

12. Forkert ND, Fiehler J, Ries T, et al. Reference-based linear curve fitting for bolus arrival time estimation in 4D MRA and MR perfusion-weighted image sequences. Magn Reson Med 2011;65:289-94

13. Willems PW, Taeshineetanakul P, Schenk B, et al. The use of 4D-CTA in the diagnostic work-up of brain arteriovenous malformations. Neuroradiology 2012;54:123-31

14. Al-Shahi R, Pal N, Lewis SC, et al. Observer agreement in the angiographic assessment of arteriovenous malformations of the brain. Stroke 2002;33:1501-08

15. Du R, Dowd CF, Johnston SC, et al. Interobserver variability in grading of brain arteriovenous malformations using the SpetzlerMartin system. Neurosurgery 2005;57:668-75, discussion 668-75

16. Iancu-Gontard D, Weill A, Guilbert F, et al. Inter- and intraobserver variability in the assessment of brain arteriovenous malformation angioarchitecture and endovascular treatment results. AJNR Am J Neuroradiol 2007;28:524-27 